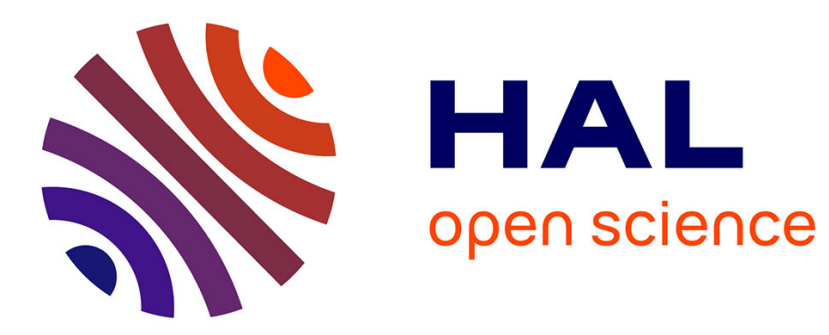

\title{
Exponential stability of a general slope limiter scheme for scalar conservation laws subject to a dissipative boundary condition
}

Dus Mathias

\section{- To cite this version:}

Dus Mathias. Exponential stability of a general slope limiter scheme for scalar conservation laws subject to a dissipative boundary condition. Mathematics of Control, Signals, and Systems, 2021. hal-03398730

\section{HAL Id: hal-03398730 \\ https://hal.science/hal-03398730}

Submitted on 23 Oct 2021

HAL is a multi-disciplinary open access archive for the deposit and dissemination of scientific research documents, whether they are published or not. The documents may come from teaching and research institutions in France or abroad, or from public or private research centers.
L'archive ouverte pluridisciplinaire HAL, est destinée au dépôt et à la diffusion de documents scientifiques de niveau recherche, publiés ou non, émanant des établissements d'enseignement et de recherche français ou étrangers, des laboratoires publics ou privés. 


\title{
Exponential stability of a general slope limiter scheme for scalar conservation laws subject to a dissipative boundary condition
}

\author{
Dus Mathias
}

Received: date / Accepted: date

\begin{abstract}
In this paper, we establish the exponential BV stability of general systems of discretized scalar conservation laws with positive speed. The focus is on numerical approximation of such systems using a wide class of slope limiter schemes built from the upwind monotone flux. The proof is based on a Lyapunov analysis taken from the continuous theory [11] and a careful use of Harten formalism.
\end{abstract}

Keywords Exponential stability, bounded variation, scalar conservation laws, flux limiter scheme.

\section{Introduction}

\subsection{Literature Review}

In this work, we are interested in the exponential stability of systems of scalar conservation laws using boundary feedback laws. The system under consideration is a set of $d$ nonlinear scalar conservation laws coupled at the boundary by a square matrix $H$ of size $d$ :

$$
\forall 1 \leq i \leq d, \begin{cases}\partial_{t} R_{i}+\partial_{x}\left[f_{i}\left(R_{i}\right)\right] & =0 \\ R_{i}(t, 0) & =[H R(t, 1)]_{i} \\ R_{i}(0, x) & =R_{i}^{0}(x) \in B V([0,1])\end{cases}
$$

where $R: \mathbb{R}^{+} \times[0,1] \mapsto \mathbb{R}^{d}\left(d \in \mathbb{N}^{*}\right), f_{i}: \mathbb{R} \mapsto \mathbb{R}$. For coherence, it is assumed that all characteristic velocities are positive and consequently, the boundary condition in (1) is adapted. In [13], the author proves the well-posedness of such system using front-tracking techniques and a classical Kruzkov analysis. The case where the sign of characteristic velocities is not fixed is out of the scope of this article. This corresponds to a problem of traffic junction treated in [3][2] just to mention a few.

Dus Mathias

Institut de mathématiques de Toulouse

E-mail: mathias.dus@math.univ-toulouse.fr 
More specifically, the focus is on the exponential stability of (1). The problem is equivalent to find sufficient conditions on $H$ such that for any $R^{0}$ initial data, the solution to (1) converges exponentially fast toward zero in the sense that

$$
\forall t \geq 0,\|R(t, .)\|_{X} \leq C e^{-\gamma t}\left\|R^{0}\right\|_{X}
$$

where $C, \gamma>0$ are constants independent on $t$ and $\|\cdot\|_{X}$ is a norm on a Banach space $X$.

In [7, Chapter 1], typical examples of systems modeled by hyperbolic PDEs with such feedback boundary conditions are cited; the telegrapher equations for electrical lines, the shallow water (Saint-Venant) equations for open channels [20], the isothermal Euler equations for gas flow in pipelines or even the Aw-Rascle equations [4] for road traffic. However it should be noted that, in previous examples, there is often an in-domain coupling which is not present in our model. Moreover, fluxes are sometimes not scalar which renders the analysis far more complicated. Hence, the family of systems we study, constitutes a simplified model for more realistic systems cited above. It introduces techniques helping in the complex studies on general systems of conservation and balance laws from [7, Chapter 1].

Additionally, the stabilization of similar systems with non-local terms receive more and more attention. We can cite [8] where the authors added a nonlocal zeroth order term to be stabilized. In this article, uncertainties on parameters and on the state of the system are allowed and an adaptive command built from an observer is designed. In [12], authors propose a spectral analysis to stabilize a scalar linear transport equation with a non-local velocity. The control is exerted at the boundary. Then, by a Lyapunov analysis they prove a local stability result for the nonlinear version of the system.

Notation: For all $R \in \mathbb{R}^{d},|R|$ designates the canonical euclidean norm of $R$. For matrices $M \in M_{d}(\mathbb{R}),|M|=\sup |M R|$. For $1 \leq p \leq \infty$ and all matrices $|R|=1, R \in \mathbb{R}^{d}$

$M \in M_{d}(\mathbb{R}),|M|_{p}$ is subordinate norm of $l^{p} . D_{d}^{+}(\mathbb{R})$ is the set of diagonal strictly positive matrices. The real $\rho_{p}(M)$ for matrices $M \in M_{d}(\mathbb{R})$ is defined by:

$$
\rho_{p}(M):=\inf _{\Delta \in D_{d}^{+}(\mathbb{R})}\left|\Delta M \Delta^{-1}\right|_{p} .
$$

Spaces $L^{p}$ on $[0,1]$ are embedded with their canonical norms $\|\cdot\|_{L^{p}}$. The space $\operatorname{Lip}_{\text {loc }}\left(\mathbb{R}^{+} ; E\right)$ corresponds to functions from $\mathbb{R}^{+}$to a Banach space $E$ which are locally Lipschitz. Let $f$ be a $C^{1}$ function from $\mathbb{R}$ to $\mathbb{R}$. Let $a, b \in \mathbb{R}$, then if $a=b$, the notation:

$$
\frac{f(b)-f(a)}{b-a}:=f^{\prime}(a)
$$

is imposed all along the article.

\subsection{Stability of the PDE system without discretization}

For the continuous PDE system, there are many results giving sufficient conditions on $H$ to ensure exponential stability. One can mention the introduction of [13] for 
a review of some of these results. The criterion which is relevant in this paper is given here:

$$
\rho_{\infty}(H):=\inf _{\Delta \in D_{d}^{+}(\mathbb{R})}\left|\Delta H \Delta^{-1}\right|_{\infty}<1 .
$$

Owing (4), we proved the exponential stability in space $X=B V([0,1])$ of systems of scalar conservation laws with positive velocities of propagation in [13]. In this work, the Banach space $X=B V([0,1])$ is also considered and the focus is on the exponential stability of the corresponding numerical solutions.

\subsection{The numerical problem}

In this paper, the focus is on finite volume approximations of system (1):

$$
\frac{R_{j}^{n+1}-R_{j}^{n}}{d t}+\frac{f_{j+1 / 2}^{n}-f_{j-1 / 2}^{n}}{d x}=0
$$

where $n, j$ are respectively the time and space index. More precisely, we find sufficient condition on $H$ such that the discretized version of (1) is exponentially stable.

The upwind flux $f_{j+1 / 2}^{n}=f\left(R_{j}^{n}\right)$ is known for its simplicity and its good properties. It is consistent and under a classic hypothesis of $C F L$, it is Total Variation Decreasing (TVD) and monotone [17] for scalar equations. These characteristics allow to prove easily that when the parameter of discretization tends towards zero, the numerical solution tends towards the unique entropy solution of the problem. For an introduction on the notion of entropy solution, we refer to [17]. However, it is over diffusive and only first order accurate. In order to correct such behavior, one can use the additional precision of a second order scheme taking:

$$
f_{j+1 / 2}^{n}=f\left(R_{j}^{n}+\tilde{R}_{j}^{n}\right)
$$

where for example $\tilde{R}_{j}^{n}=\frac{R_{j+1}^{n}-R_{j}^{n}}{d x} \frac{d x}{2}$. However, a second order scheme cannot be TVD by Godunov Theorem and have oscillatory behaviors around discontinuities. Moreover, it can also be unstable as it is the case for the example cited just above. This is why one introduces a slope limiter $\phi[23]$ whose role is to damp the effect of the second order flux around discontinuities. The slope limiter scheme is defined taking:

$$
\tilde{R}_{j}:=\phi\left(\frac{R_{j}^{n}-R_{j-1}^{n}}{d x}, \frac{R_{j+1}^{n}-R_{j}^{n}}{d x}\right) \frac{d x}{2}
$$

where $\phi$ is called the slope limiter that will be defined carefully later. The main contribution of the paper is to find sufficient condition on $\phi$ and $H$ to prove the exponential $B V([0,1])$ stability of the numerical solution built from the slope limiter scheme giving an explicit formula for the dissipation rate.

There are several results for the stabilization of discretized 1D wave equation. A very instructive survey from Trélat [30] gives an insight of techniques for the stabilization of discretized hyperbolic PDEs. One can also cite [29] where the authors prove the boundary stabilization of a semi-discretized 1D wave equation. Adding a numerical viscosity term damping high frequencies, they prove a uniform 
exponential stability decay of the energy uniformly with respect to the parameter of discretization. The article [28] deals with the case of an in-domain damping.

The main concern is to obtain a uniform observability inequality whose constant is independent on the parameter of discretization. This is not an easy task since the observability of the continuous system does not necessarily imply the same for the fully discrete or the semi discrete system. The survey [33] summarizes most of the main results about this question. The main problem comes from high frequency modes and their group velocity. The group velocity of two consecutive high frequency modes tends to zero as the discretization parameter goes to zero which prevent from using Ingham's inequality. This makes the observation (at a finite fixed time) very difficult as the discretization get finer [24]. This is why it is primordial to add a non physical numerical viscosity in order to damp these high frequencies.

For completeness, we cite [15] where the author prove the uniform exponential decay for another semi-discretized scheme simulating an in-domain damped wave equation. The case of semilinear wave equation is treated in [1]. The method to deduce exponential stabilization for the full discretized case is given in [16].

However to the author's knowledge, few results are known about the stabilization of discretized transport equations of the form (1). The method presented here is very different from observation inequalities techniques. We rather use discrete Lyapunov functions to prove exponential decay of our solution. In a $L^{2}$ framework, the articles $[18,5]$ prove a stabilization result for discretized linear balance laws coupled in the domain and at the boundary using such techniques. However, it does not seem to be generalizable to a $B V$ context for nonlinear scalar conservation laws. In section 3, we address this problem introducing a discrete Lyapunov functional in BV.

\subsection{Problem setting}

For wellposedness and scheme convergence results, we consider a more general boundary condition:

$$
\begin{cases}\partial_{t} R+\partial_{x}[f(R)] & =0 \\ R(t, 0) & =g(R(t, 1)) \\ R(0, x) & =R^{0}(x) \in B V([0,1]) .\end{cases}
$$

where the following hypothesis on $f$ and $g$ hold:

$-f$ is diagonal in the sense that:

$$
\forall 1 \leq i \leq d, R \in \mathbb{R}^{d}, f_{i}(R)=f_{i}\left(R_{i}\right) .
$$

Moreover, all the $f_{i}$ seen as functions from $\mathbb{R}$ to $\mathbb{R}$ are non decreasing. Doing so, the boundary condition of (5) has sense.

- There exist two positive constants $0<v_{\min }<v_{\max }$ such that for all $1 \leq i \leq d$,

$$
\forall(u, v) \in \mathbb{R}^{2}, v_{\min }|u-v| \leq\left|f_{i}(u)-f_{i}(v)\right| \leq v_{\max }|u-v| .
$$

- The boundary feedback function $g: \mathbb{R}^{d} \mapsto \mathbb{R}^{d}$ is Lipschitz with Lipschitz constant $L_{g}>0$. Moreover, we impose $g(0)=0$. 
Remark 1 The components of a solution $R$ to (5) interact with each others only at the boundary.

In this paper, the framework is $B V([0,1])$. This space is embedded with its classical norm $\|\cdot\|_{B V([0,1])}$ defined by

$$
\forall R \in B V([0,1]),\|R\|_{B V([0,1])}=T V_{[0,1]}(R)+\|R\|_{L^{1}([0,1])}
$$

where:

$$
T V(R)=\sup _{n,\left(x_{1}, \ldots, x_{n}\right)}\left\{\sum_{i=1}^{n-1}\left|R\left(x_{i+1}\right)-R\left(x_{i}\right)\right|\right\} .
$$

The reason why the BV space is considered is because any function with bounded variations has a left limit and a right limit at each point $x$ of $[0,1]$. Hence, it is easy to define the trace operator and impose a boundary condition. Moreover, $B V([0,1])$ has a very interesting property of compactness which will be very useful when we will pass to the limit when the parameter of discretization tends towards zero. These properties are summed up in a lemma and a theorem (see references [9, Lemma 2.1, Theorem 2.4]) :

Lemma 1 Let $R:[0,1] \mapsto \mathbb{R}^{d}$ with bounded variations. Then for all $x \in(0,1)$, the left and right limit

$$
R\left(x^{-}\right)=\lim _{y \rightarrow x^{-}} R(y), \quad R\left(x^{+}\right)=\lim _{y \rightarrow x^{+}} R(y)
$$

exist. Moreover, $R\left(0^{+}\right)$and $R\left(1^{-}\right)$are also well defined and $R$ has at most countably many point of discontinuities.

The following theorem is from Helly and states the compactness of $B V([0,1])$ in $L_{l o c}^{1}\left(\mathbb{R}^{+}, L^{1}([0,1])\right)$.

Theorem 1 Let $\left(R_{\nu}\right)_{\nu}$ be a sequence of functions from $\mathbb{R}^{+} \times[0,1]$ to $\mathbb{R}^{d}$ such that there exists constants $C, M$ and $L$ satisfying

$$
\forall \nu>1, x \in[0,1], t \geq 0, T V_{[0,1]}\left(R_{\nu}(t, .)\right) \leq C,\left|R_{\nu}(t, x)\right| \leq M
$$

and

$$
\forall 0 \leq t, s \leq T,\left\|R_{\nu}(t, .)-R_{\nu}(s, .)\right\|_{L^{1}([0,1])} \leq L|t-s| .
$$

Then there exists a subsequence $\left(R_{\mu}\right)_{\mu}$ converging toward a certain $R$ in $L_{\text {loc }}^{1}\left(\mathbb{R}^{+}, L^{1}([0,1])\right)$ and this limit satisfies $(8)-(9)$ with $R_{\nu}$ replaced by $R$.

When the initial data is supposed to be $L^{\infty}$ only, an entropic solution exists and is unique [22]. Moreover, it is possible to define the trace in a certain sense $[26,32]$. However, the method we use to prove exponential stability crucially uses the $B V$ hypothesis and this is why the $L^{\infty}$ framework is out of the scope of this paper. 


\subsection{Outline}

The article is organized as follows. In section 2 , we precisely define the slope limiter scheme and prove the convergence in $B V([0,1])$ of the corresponding numerical solution. As a by-product, a well-posedness result for system (5) is proved. In section 3, the framework is restricted to linear boundary feedback operator $g(R)=$ $H R$. Then, the exponential stability of the scheme in $B V([0,1])$ is proved giving an explicit formula for the dissipation rate. Passing to the limit, we give another proof of the exponential stability of the continuous system which was already stated in [13]. In section 4, simulations are given to illustrate results from section 3. A study of saturated controls is also given. Finally, the conclusions and perspectives are exposed in the last part of this work.

\section{Well-posedness and numerical approximation results}

In this section, we introduce rigorously the slope limiter scheme corresponding to (5) and prove the convergence of such a scheme. Firstly, let us consider a space step $d x=1 / N\left(N \in \mathbb{N}^{*}\right)$ and a time step $d t>0$ such that the following CFL condition holds (recall the definition of $v_{\max }$ in $(6)$ ):

$$
v_{\max } \nu \leq 1-\xi
$$

with

$$
\nu:=\frac{d t}{d x}
$$

and where $\xi$ is a real number such that:

$$
0<\xi<1
$$

Doing so, the space-time mesh is given by:

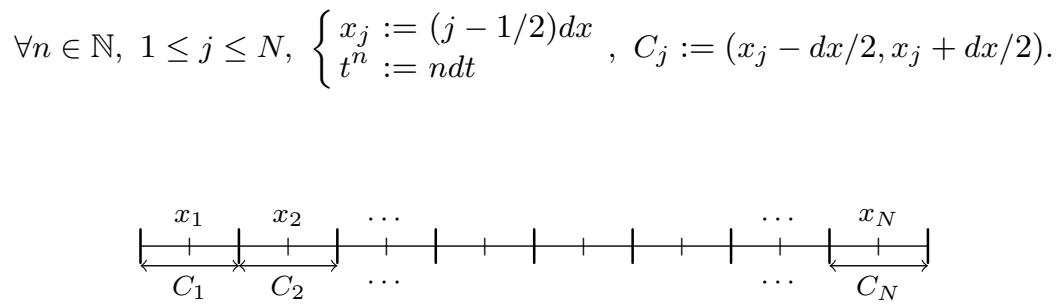

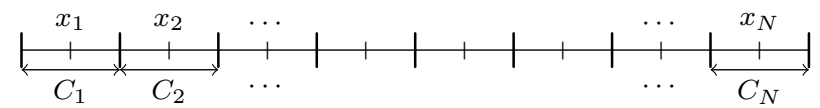

Fig. 1: The space grid

All along this paper, the superscript $n$ is exclusively allocated to designate time indices. Whereas, subscripts $i$ and $j$ are the indices corresponding respectively 
to the vector component and space grid. The numerical approximation $R_{\Delta x}^{0}$ of $R^{0} \in B V([0,1])$ is given below

$$
\forall 1 \leq j \leq N, x \in C_{j}, R_{\Delta x}^{0}(x)=\frac{1}{d x} \int_{C_{j}} R^{0}(x) d x .
$$

In our context, the definition of a four point finite volume scheme is given below:

Definition 1 A four-point finite volume scheme is defined a follows:

- The scheme is initialized defining $\left(R_{j}^{0}\right)_{1 \leq j \leq N}$ as in (11).

- Then the numerical solution is built by induction. For $n \in \mathbb{N},\left(R_{j}^{n}\right)_{1 \leq j \leq N}$ is supposed to be given. We define $\left(R_{j}^{n+1}\right)_{1 \leq j \leq N}$ using the following relation

$$
\forall 2 \leq j \leq N-1, \frac{R_{j}^{n+1}-R_{j}^{n}}{d t}+\frac{f_{j+1 / 2}^{n}-f_{j-1 / 2}^{n}}{d x}=0
$$

where $f_{j+1 / 2}^{n}$ is the approximation of the flux at the right interface of cell $j$ :

$$
\forall 1 \leq j \leq N-1, f_{j+1 / 2}^{n}=\tilde{f}\left(R_{j-1}^{n}, R_{j}^{n}, R_{j+1}^{n}\right) .
$$

The function $\tilde{f}$ is called the three point approximation of the flux $f$. The choice of such a function will be given later with careful justifications.

- At the boundary the scheme degenerates into the upwind one:

$$
\left\{\begin{array}{l}
\frac{R_{N}^{n+1}-R_{N}^{n}}{d t}+\frac{f\left(R_{N}^{n}\right)-f\left(R_{N-1}^{n}\right)}{d x}=0 \\
\frac{R_{1}^{n+1}-R_{1}^{n}}{d t}+\frac{f\left(R_{1}^{n}\right)-f\left(R_{0}^{n}\right)}{d x}=0 \\
R_{0}^{n}:=g\left(R_{N}^{n}\right) .
\end{array}\right.
$$

There are many possibilities for the choice of the function $\tilde{f}$. For example, one can take:

$$
\tilde{f}\left(R_{j-1}^{n}, R_{j}^{n}, R_{j+1}^{n}\right)=f\left(R_{j}^{n}\right) .
$$

It corresponds to the classic upwind scheme when the transport velocity is positive. Recalling what was said in the introduction, the first main advantage of such scheme is that it is TVD and consistent allowing convergence towards a weak solution to (5). The other advantage is that it is monotone forcing the scheme to converge towards the unique entropy solution of (5). However, it is known for its diffusivity giving poor results in simulation. This is why one introduces the slope limiter flux [31] [23]:

$$
\tilde{f}\left(R_{j-1}^{n}, R_{j}^{n}, R_{j+1}^{n}\right)=f\left(R_{j}^{n}+\tilde{R}_{j}^{n}\right)
$$

where the term $\tilde{R}_{j}^{n}$ is given by a piecewise linear approximation of the solution:

$$
\forall 1 \leq i \leq d, \tilde{R}_{i, j}^{n}=\phi\left(\frac{R_{i, j}^{n}-R_{i, j-1}^{n}}{d x}, \frac{R_{i, j+1}^{n}-R_{i, j}^{n}}{d x}\right) \frac{d x}{2}
$$

where $\phi$ is assumed to be of the form: 


$$
\phi(u, v)= \begin{cases}0 & \text { if } v=0 \\ \phi_{r}(u / v) \psi_{d x}(v) & \text { otherwise }\end{cases}
$$

such that there exists $0 \leq \beta<1 / 2$ such that:

$$
\left|\psi_{d x}(v)\right| \leq v \text { and }\left|\psi_{d x}(v)\right| \leq d x^{-\beta}
$$

and $\phi_{r}: \mathbb{R} \rightarrow \mathbb{R}$ and for all $r \leq 0, \phi_{r}(r)=0$.

The natural choice for $\psi_{d x}$ is:

$$
\psi_{d x}(v)= \begin{cases}v & \text { if }|v| \leq d x^{-\beta} \\ \operatorname{sign}(v) d x^{-\beta} & \text { otherwise. }\end{cases}
$$

Hence, if $R_{i, j+1}^{n}-R_{i, j}^{n} \neq 0$, then:

$$
\tilde{R}_{i, j}^{n}:=\phi_{r}\left(r_{i, j+1 / 2}^{n}\right) \psi_{d x}\left(\frac{R_{j+1}^{n}-R_{j}^{n}}{d x}\right) \frac{d x}{2} .
$$

The variable $r_{i, j+1 / 2}^{n} \in \mathbb{R}^{d}$ is defined by:

$$
\forall 1 \leq i \leq d, r_{i, j+1 / 2}^{n}:=\frac{R_{i, j}^{n}-R_{i, j-1}^{n}}{R_{i, j+1}^{n}-R_{i, j}^{n}} .
$$

Remark 2 When the flux is linear, slope limiter schemes and flux limiter schemes coincide [23, p. 114].

The slope limiter $\phi$ is designed such that when the solution is regular i.e $r_{j+1 / 2} \approx 1$, the flux $\tilde{f}$ is closed to a second order flux. On the contrary, when the solution is not regular $\left(r_{j+1 / 2} \gg 1\right)$ or admits a local extrema $\left(r_{j+1 / 2} \leq 0\right)$, the second order scheme gives poor oscillatory results. In this case, we prefer to give more weight on the upwind TVD scheme imposing $\phi \approx 0$. We refer to [23, Section 6.6-6.9] for a more complete description of how slope limiter schemes were designed.

We define the following coefficient for all $1 \leq i \leq d, 1 \leq j \leq N-1$, which will be useful all along this article:

$$
\begin{aligned}
& a_{i, j-1}^{n}:=\nu \frac{f_{i}\left(R_{i, j}^{n}+\tilde{R}_{i, j}^{n}\right)-f_{i}\left(R_{i, j-1}^{n}+\tilde{R}_{i, j-1}^{n}\right)}{R_{i, j}^{n}+\tilde{R}_{i, j}^{n}-R_{i, j-1}^{n}-\tilde{R}_{i, j-1}^{n}} \\
& \times\left(1+\frac{\phi_{r}\left(r_{i, j+1 / 2}^{n}\right)}{2 r_{i, j+1 / 2}^{n}} \frac{\psi_{d x}\left(\frac{R_{i, j+1}^{n}-R_{i, j}^{n}}{d x}\right)}{\frac{R_{i, j+1}^{n}-R_{i, j}^{n}}{d x}}-\frac{\phi_{r}\left(r_{i, j-1 / 2}^{n}\right)}{2} \frac{\psi_{d x}\left(\frac{R_{i, j}^{n}-R_{i, j-1}^{n}}{d x}\right)}{\frac{R_{i, j}^{n}-R_{i, j-1}^{n}}{d x}}\right) .
\end{aligned}
$$

For the pathologic cases, one defines $a_{i, j-1}^{n}$ as follows:

- If $R_{i, j}^{n}-R_{i, j-1}^{n}=0$ (ie $r_{i, j+1 / 2}^{n}=0$ and $r_{i, j-1 / 2}^{n}$ ill-defined), then $a_{i, j-1}^{n}:=$ $\nu f_{i}^{\prime}\left(R_{i, j}^{n}\right)$

- Otherwise, if $R_{i, j}^{n}-R_{i, j-1}^{n} \neq 0$ and $R_{i, j+1}^{n}-R_{i, j}^{n}=0\left(r_{i, j+1 / 2}^{n}\right.$ is ill-defined $)$, the coefficient $\frac{\phi_{r}\left(r_{i, j+1 / 2}^{n}\right)}{2 r_{i, j+1 / 2}^{n}} \frac{\psi_{d x}\left(\frac{R_{i, j+1}^{n}-R_{i, j}^{n}}{d x}\right)}{\frac{R_{i, j+1}^{n}-R_{i, j}^{n}}{d x}}$ is replaced by zero in (18). 
- If $R_{i, j}^{n}+\tilde{R}_{i, j}^{n}-R_{i, j-1}^{n}-\tilde{R}_{i, j-1}^{n}=0$, then recall that $\frac{f_{i}\left(R_{i, j}^{n}+\tilde{R}_{i, j}^{n}\right)-f_{i}\left(R_{i, j-1}^{n}+\tilde{R}_{i, j-1}^{n}\right)}{R_{i, j}^{n}+\tilde{R}_{i, j}^{n}-R_{i, j-1}^{n}-\tilde{R}_{i, j-1}^{n}}=$ $f_{i}^{\prime}\left(R_{i, j}^{n}+\tilde{R}_{i, j}^{n}\right)$ by the convention of notation (3).

For $j=N$, we set:

$$
a_{i, N-1}^{n}:=\nu \frac{f^{i}\left(R_{i, N}^{n}\right)-f^{i}\left(R_{i, N-1}^{n}\right)}{R_{i, N}^{n}-R_{i, N-1}^{n}} .
$$

The diagonal matrix with $a_{i, j}^{n}$ as entries is denoted $A_{j}^{n}:=\operatorname{diag}\left\{a_{i, j}^{n} \mid 1 \leq i \leq d\right\}$. Hence, the scheme can be reformulated under an upwind form:

$$
R_{j}^{n+1}=R_{j}^{n}-A_{j-1}^{n}\left(R_{j}^{n}-R_{j-1}^{n}\right)
$$

In order to ensure that our scheme is TVD, we impose the following condition of the slope limiter $\phi$ :

Hypothesis 1 There exists $0 \leq v_{\text {num }} \leq v_{\min }$ such that:

$$
0 \leq \phi_{r}(r) \leq \min \left\{2\left(\frac{1}{\nu v_{\max }}-1\right) r, 2\left(1-\frac{v_{\text {num }}}{v_{\min }}\right)\right\}
$$

This hypothesis is fundamental to ensure Harten's condition [19]:

Lemma 2 If Hypothesis 1 is satisfied, then Harten's condition is satisfied:

$$
\nu v_{n u m} \leq a_{n, j}^{i} \leq 1
$$

Proof Looking at (18), one has:

$$
\begin{aligned}
& a_{i, j}^{n} \leq \nu v_{\max }\left(1+\frac{\phi_{r}\left(r_{i, j+1 / 2}^{n}\right)}{2 r_{i, j+1 / 2}^{n}}\right), \\
& a_{i, j}^{n} \geq \nu v_{\min }\left(1-\frac{\phi_{r}\left(r_{i, j-1 / 2}^{n}\right)}{2}\right) .
\end{aligned}
$$

By Hypothesis 1:

$$
\begin{aligned}
\nu v_{\max }\left(1+\frac{\phi_{r}\left(r_{i, j+1 / 2}^{n}\right)}{2 r_{i, j+1 / 2}^{n}}\right) & \leq 1 \\
\nu v_{\min }\left(1-\frac{\phi_{r}\left(r_{i, j-1 / 2}^{n}\right)}{2}\right) & \geq \nu v_{n u m}
\end{aligned}
$$

which immediately gives the result of the Lemma.

Before going into the main result of this section, we introduce a new notation. Let $R_{\Delta x}$ be the piecewise constant function equal to $R_{j}^{n}$ on each cell $[n d t,(n+1) d t] \times C_{j}$. This numerical approximation helps us proving the existence of a solution to (5). Meanwhile, Kruzhkov theory allows to prove the uniqueness. To prove the following Theorem 2, techniques are quite classical and plenty of works deal with the convergence of slope limiter schemes for scalar conservation laws in several dimensions and when the domain is the whole real line [10] [21] [25]. In our case, this is a bit more complex since we have to take into account the boundary condition. 
Theorem 2 System (5) admits a unique entropy solution $R \in L_{l o c}^{\infty}\left(\mathbb{R}^{+}, B V([0,1])\right) \cap$ $\operatorname{Lip}_{\text {loc }}\left(\mathbb{R}^{+}, L^{1}([0,1])\right)$ in the sense that for all $T>0$ :

- The entropy decay estimate holds:

$$
\forall k \in \mathbb{R}^{d}, \sum_{i=1}^{d} \int_{0}^{T} \int_{0}^{1}\left\{\left|R_{i}-k_{i}\right| \partial_{t} \varphi_{i}+\left(f_{i}\left(R_{i}\right)-f_{i}\left(k_{i}\right)\right) \operatorname{sign}\left(R_{i}-k_{i}\right) \partial_{x} \varphi_{i}\right\} d x d t \geq 0
$$

for all $\varphi_{i} \geq 0$ and $\varphi_{i} \in C_{c}^{1}(] 0, T[\times] 0,1[)$.

$-R(0,)=.R^{0}$ in the almost everywhere sense.

$-R\left(., 0^{+}\right)=g\left(R\left(., 1^{-}\right)\right)$in the almost everywhere sense.

Moreover, the following convergence properties hold

$$
\begin{gathered}
\forall t \geq 0, d t>0, T V_{[0,1]}(R(t, .)) \leq \limsup _{N \rightarrow+\infty} \sup _{s \in[t, t+d t]} T V_{[0,1]}\left(R_{\Delta x}(s, .)\right) . \\
\forall t \geq 0, \lim _{N \rightarrow \infty}\left\|R(t, .)-R_{\Delta x}(t, .)\right\|_{L^{1}([0,1])}=0 .
\end{gathered}
$$

Proof The proof is given in Appendix A.

\section{Exponential BV stability}

In this section, we focus on a particular case of system of (5).

$$
\begin{cases}\partial_{t} R+\partial_{x}[f(R)] & =0 \\ R(t, 0) & =H R(t, 1) \\ R(0, x) & =R^{0}(x) \in B V([0,1])\end{cases}
$$

where $H \in M_{d}(\mathbb{R})$. We introduce the following stability hypothesis.

Hypothesis 2 The feedback matrix $H$ satisfies:

$$
\rho_{1}(H)<1
$$

Remark 3 By [11, Remark 1.4]

$$
\forall M \in M_{d}(\mathbb{R}), \rho_{1}(H)=\rho_{\infty}(H)
$$

so that Hypothesis 2 can also be written as:

$$
\rho_{\infty}(H)<1
$$

The following Lyapunov functional is proposed for functions which are constant on the space mesh. 
Definition 2 Let $N$ be in $\mathbb{N}^{*}, \gamma>0$ and $P \in D_{d}^{+}(\mathbb{R})$. For any function $R$ piecewise constant on cells $C_{j}$ taking its values in $\mathbb{R}^{d}$, the $B V$ Lyapunov functional $\mathcal{L}$ is given by

$$
\mathcal{L}(R)=\sum_{i=1}^{d} P_{i} \sum_{j=0}^{N-1}\left|R_{i, j+1}-R_{i, j}\right| e^{-\gamma x_{j}}
$$

where

$$
x_{0}:=-d x / 2, R_{0}:=H R_{N} .
$$

Next lemma ensures the equivalence between $\mathcal{L}$ and $\|\cdot\|_{B V([0,1])}$ defined in (7).

Lemma 3 Suppose Hypothesis 2. For all $P \in D_{d}^{+}\left(\mathbb{R}^{+}\right)$such that $\left|P H P^{-1}\right|_{1} \leq 1$ and for all $\gamma>0$, there exists a constant $C(P, \gamma, H)>1$ such that for all $N \in \mathbb{N}^{*}$ and all functions $R$ piecewise constant on cells $\left(C_{j}\right)_{j}$ :

$$
\frac{\mathcal{L}(R)}{C(P, \gamma, H)} \leq\|R\|_{B V([0,1])} \leq C(P, \gamma, H) \mathcal{L}(R)
$$

Proof We define the extension $\tilde{R}$ of $R$ at $C_{0}:=(-d x, 0)$ setting $\tilde{R}(x)=H R(1)$ on $C_{0}$. In [13, Lemma 2], it is proved that:

$$
\frac{\mathcal{L}(R)}{C(P, \gamma, H)} \leq T V(\tilde{R})+\|\tilde{R}\|_{L^{1}([0,1])} \leq C(P, \gamma, H) \mathcal{L}(R) .
$$

Additionally, as $\tilde{R}$ is an extension on the left boundary of $R$ and by the definition of the $B V$ norm, it holds:

$$
\|R\|_{B V([0,1])} \leq T V(\tilde{R})+\|\tilde{R}\|_{L^{1}([0,1])}=\|R\|_{B V([0,1])}+\left|R_{1}-\tilde{R}_{0}\right|+\left|\tilde{R}_{0}\right| d x .
$$

As $\tilde{R}_{0}=H R_{N}$ by definition and as $\|\cdot\|_{L^{\infty}([0,1])} \leq C\|\cdot\|_{B V([0,1])}(C>0$ is a constant depending on the parameters of the problem only), one has:

$$
\|R\|_{B V([0,1])} \leq T V(\tilde{R})+\|\tilde{R}\|_{L^{1}([0,1])} \leq C(H)\|R\|_{B V([0,1])} .
$$

Hence, using (25), it holds:

$$
\frac{\mathcal{L}(R)}{C(P, \gamma, H)} \leq\|R\|_{B V([0,1])} \leq C(P, \gamma, H) \mathcal{L}(R)
$$

where we may have changed the constant $C(P, \gamma, H)$. The lemma is proved.

The reason why we introduce a discrete Lyapunov functional is because we cannot deduce the exponential stability of the numerical solution from the stability of the "continuous" one proven in [13]. Indeed, the convergence of the numerical solution towards the continuous solution is only proven to be in $L_{l o c}^{1}\left(\mathbb{R}^{+} ; L^{1}([0,1])\right)$ in general [17, Theorem 5.2]. This is not enough to prove an exponential stability result even in $L^{1}([0,1])$. This is why, we need to study the stability property of the scheme in itself introducing a discrete Lyapunov functional. The main result of this paper is given in the following theorem whose proof is the object of the section. 
Theorem 3 Under Hypothesis 1-2 and for all $\gamma>0,0 \leq c<v_{n u m}, P \in D_{d}^{+}\left(\mathbb{R}^{d}\right)$ such that $\left|P H P^{-1}\right|_{1}<e^{-\gamma}$, there exists $\varepsilon$ such that if $\gamma d t<\varepsilon$, the discrete Lyapunov estimate holds:

$$
\forall n \geq 0, \quad \mathcal{L}\left(R^{n}\right) \leq e^{-c \gamma n d t} \mathcal{L}\left(R^{0}\right)
$$

To understand the consequences of Theorem 3, we consider a limiter $\phi_{r}$ satisfying Hypothesis 1 with $v_{n u m}=\alpha v_{\min }$ and $\alpha \in[0,1]$. Then, a Sweby diagram [27] is drawn in Figure 2 where the colored region represents the zone corresponding to Hypothesis 1 . Theorem 3 states that for such $\phi_{r}$ the dissipation rate of the numerical solution is bounded from below by $\alpha v_{\text {min }}\left(=v_{n u m}\right)$. Remark that the best estimation $v_{\text {num }}=v_{\text {min }} \Longleftrightarrow \alpha=1$ is obtained for only one scheme $\phi_{r}=0$ which is the upwind scheme.

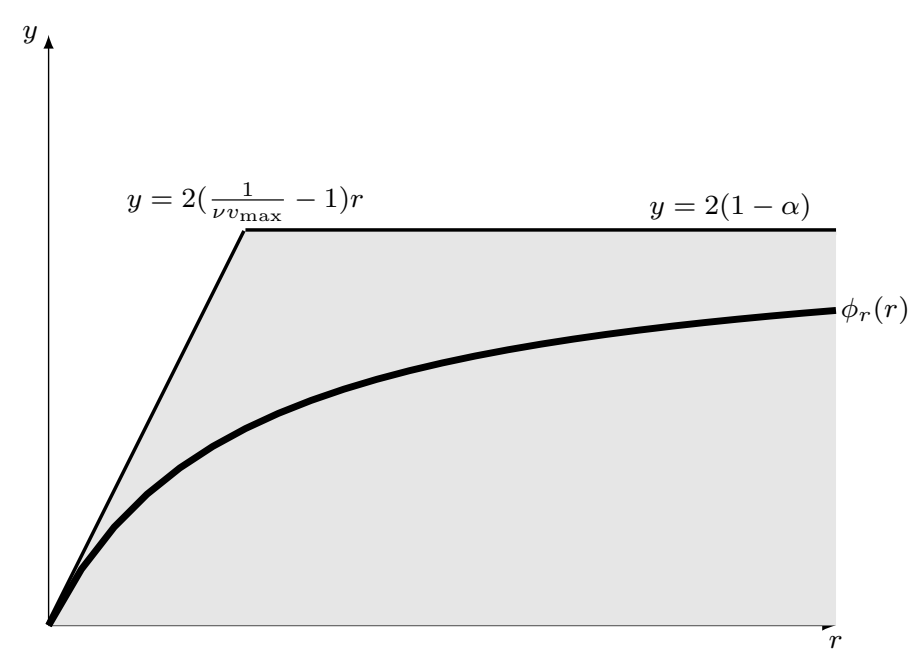

Fig. 2: The Sweby diagram

Proof Let $N, n$ be positive integers. By the definition of $\mathcal{L}$, we have:

$$
\mathcal{L}\left(R^{n+1}\right)=\sum_{i=1}^{d} P_{i} \sum_{j=0}^{N-1}\left|R_{i, j+1}^{n+1}-R_{i, j}^{n+1}\right| e^{-\gamma x_{j}} .
$$

The $i$ th component of the Lyapunov functional $\mathcal{L}$ is given here:

$$
\mathcal{L}_{i}\left(R^{n+1}\right):=\sum_{j=0}^{N-1}\left|R_{i, j+1}^{n+1}-R_{i, j}^{n+1}\right| e^{-\gamma x_{j}} .
$$

For $1 \leq i \leq d$ and $1 \leq j \leq N-1$, we estimate the intensity of the discontinuity $R_{i, j+1}^{n+1}-\bar{R}_{i, j}^{n+\overline{1}}$. 


$$
R_{i, j+1}^{n+1}-R_{i, j}^{n+1}=\left(1-a_{i, j}^{n}\right)\left(R_{i, j+1}^{n}-R_{i, j}^{n}\right)+a_{i, j-1}^{n}\left(R_{i, j}^{n}-R_{i, j-1}^{n}\right)
$$

where $a_{j}^{n}$ was defined in (18). Because of the Harten's condition (19) we have for $1 \leq i \leq d$ and $1 \leq j \leq N-1$ :

$$
\left|R_{i, j+1}^{n+1}-R_{i, j}^{n+1}\right| \leq\left(1-a_{i, j}^{n}\right)\left|R_{i, j+1}^{n}-R_{i, j}^{n}\right|+a_{i, j-1}^{n}\left|R_{i, j}^{n}-R_{i, j-1}^{n}\right| .
$$

Multiplying by $e^{-\gamma x_{j}}$ and summing over $1 \leq j \leq N-1$, we get

$$
\begin{aligned}
\sum_{j=1}^{N-1}\left|R_{i, j+1}^{n+1}-R_{i, j}^{n+1}\right| e^{-\gamma x_{j}} \leq & \sum_{j=1}^{N-2}\left(1-a_{i, j}^{n}\left(1-e^{-\gamma d x}\right)\right)\left|R_{i, j+1}^{n}-R_{i, j}^{n}\right| e^{-\gamma x_{j}} \\
& +\left(1-a_{i, N-1}^{n}\right)\left|R_{i, N}^{n}-R_{i, N-1}^{n}\right| e^{-\gamma x_{N-1}} \\
& +a_{i, 0}^{n}\left|R_{i, 1}^{n}-R_{i, 0}^{n}\right| e^{-\gamma d x / 2} .
\end{aligned}
$$

Coming back to the Lyapunov functional $\mathcal{L}_{i}$, it holds:

$$
\begin{aligned}
\mathcal{L}_{i}\left(R^{n+1}\right) \leq & \sum_{j=1}^{N-2}\left(1-a_{i, j}^{n}\left(1-e^{-\gamma d x}\right)\right)\left|R_{i, j+1}^{n}-R_{i, j}^{n}\right| e^{-\gamma x_{j}} \\
& +\left(1-a_{i, N-1}^{n}\right)\left|R_{i, N}^{n}-R_{i, N-1}^{n}\right| e^{-\gamma x_{N-1}} \\
& +a_{i, 0}^{n}\left|R_{i, 1}^{n}-R_{i, 0}^{n}\right| e^{-\gamma d x / 2} \\
& +\left|R_{i, 1}^{n+1}-R_{i, 0}^{n+1}\right| e^{\gamma d x / 2} .
\end{aligned}
$$

Now, we estimate $\left|R_{i, 1}^{n+1}-R_{i, 0}^{n+1}\right|$ :

$$
\begin{aligned}
R_{i, 1}^{n+1}-R_{i, 0}^{n+1} & =R_{i, 1}^{n}+a_{i, 0}^{n}\left(R_{i, 0}^{n}-R_{i, 1}^{n}\right)-R_{i, 0}^{n+1} \\
& =\left(R_{i, 1}^{n}-R_{i, 0}^{n}\right)\left(1-a_{i, 0}^{n}\right)+R_{i, 0}^{n}-R_{i, 0}^{n+1} .
\end{aligned}
$$

Taking the absolute value and by triangle inequality, one obtains:

This gives:

$$
\left|R_{i, 1}^{n+1}-R_{i, 0}^{n+1}\right| \leq\left(1-a_{i, 0}^{n}\right)\left|R_{i, 1}^{n}-R_{i, 0}^{n}\right|+\left|R_{i, 0}^{n}-R_{i, 0}^{n+1}\right| .
$$

$$
\begin{aligned}
\mathcal{L}_{i}\left(R^{n+1}\right) \leq & \sum_{j=1}^{N-2}\left(1-a_{i, j}^{n}\left(1-e^{-\gamma d x}\right)\right)\left|R_{i, j+1}^{n}-R_{i, j}^{n}\right| e^{-\gamma x_{j}} \\
& +\left(1-a_{i, N-1}^{n}\right)\left|R_{i, N}^{n}-R_{i, N-1}^{n}\right| e^{-\gamma x_{N-1}} \\
& +\left(1-a_{i, 0}^{n}\left(1-e^{-\gamma d x}\right)\right)\left|R_{i, 1}^{n}-R_{i, 0}^{n}\right| e^{\gamma d x / 2} \\
& +\left|R_{i, 0}^{n+1}-R_{i, 0}^{n}\right| e^{\gamma d x / 2}
\end{aligned}
$$

which is no more than:

$$
\begin{aligned}
\mathcal{L}_{i}\left(R^{n+1}\right) \leq & \sum_{j=0}^{N-2}\left(1-a_{i, j}^{n}\left(1-e^{-\gamma d x}\right)\right)\left|R_{i, j+1}^{n}-R_{i, j}^{n}\right| e^{-\gamma x_{j}} \\
& +\left(1-a_{i, N-1}^{n}\right)\left|R_{i, N}^{n}-R_{i, N-1}^{n}\right| e^{-\gamma x_{N-1}} \\
& +\left|R_{i, 0}^{n+1}-R_{i, 0}^{n}\right| e^{\gamma d x / 2} .
\end{aligned}
$$


Using the fact that there exists $\varepsilon$ such that for $\gamma d x \leq \varepsilon, 1-e^{-\gamma d x} \geq \frac{c}{v_{\text {num }}} \gamma d x$ (because $\frac{c}{v_{\text {num }}}<1$ by assumption), we get:

$$
\begin{aligned}
\mathcal{L}_{i}\left(R^{n+1}\right) \leq & \sum_{j=0}^{N-2}\left(1-a_{i, j}^{n} \frac{c \gamma d x}{v_{n u m}}\right)\left|R_{i, j+1}^{n}-R_{i, j}^{n}\right| e^{-\gamma x_{j}} \\
& +\left(1-a_{i, N-1}^{n}\right)\left|R_{i, N}^{n}-R_{i, N-1}^{n}\right| e^{-\gamma x_{N-1}} \\
& +\left|R_{i, 0}^{n+1}-R_{i, 0}^{n}\right| e^{\gamma d x / 2} .
\end{aligned}
$$

Then we add and subtract $\left(1-a_{i, N-1}^{n} \frac{c \gamma d x}{v_{n u m}}\right)\left|R_{i, N}^{n}-R_{i, N-1}^{n}\right| e^{-\gamma(N-1) d x}$ in the first and second line respectively to get:

$$
\begin{aligned}
\mathcal{L}_{i}\left(R^{n+1}\right) \leq & \sum_{j=0}^{N-1}\left(1-a_{i, j}^{n} \frac{c \gamma d x}{v_{n u m}}\right)\left|R_{i, j+1}^{n}-R_{i, j}^{n}\right| e^{-\gamma x_{j}} \\
& -a_{i, N-1}^{n}\left(1-\frac{c \gamma d x}{v_{n u m}}\right)\left|R_{i, N}^{n}-R_{i, N-1}^{n}\right| e^{-\gamma x_{N-1}} \\
& +\left|R_{i, 0}^{n+1}-R_{i, 0}^{n}\right| e^{\gamma d x / 2} .
\end{aligned}
$$

Finally, using the fact that $a_{N-1}^{n}\left(R_{N}^{n}-R_{N-1}^{n}\right)=-\left(R_{N}^{n+1}-R_{N}^{n}\right)$ and the fact that $1-a_{i, j}^{n} \frac{c \gamma d x}{v_{\text {num }}} \leq 1-c \gamma d t$ (see $\left.(19)\right)$, the estimate of $\mathcal{L}_{i}$ writes:

$$
\begin{aligned}
\mathcal{L}_{i}\left(R^{n+1}\right) \leq & (1-c \gamma d t) \mathcal{L}_{i}\left(R^{n}\right) \\
& -\left(1-\frac{c \gamma d x}{v_{\text {num }}}\right)\left|R_{i, N}^{n+1}-R_{i, N}^{n}\right| e^{-\gamma x_{N-1}} \\
& +\left|R_{i, 0}^{n+1}-R_{i, 0}^{n}\right| e^{\gamma d x / 2} .
\end{aligned}
$$

Using the discrete boundary condition (14), one changes the last boundary term:

$$
\begin{aligned}
\mathcal{L}_{i}\left(R^{n+1}\right) \leq & (1-c \gamma d t) \mathcal{L}_{i}\left(R^{n}\right) \\
& -\left(1-\frac{c \gamma d x}{v_{n u m}}\right)\left|R_{i, N}^{n+1}-R_{i, N}^{n}\right| e^{-\gamma x_{N-1}} \\
& +\left|\left[H R_{N}^{n+1}\right]_{i}-\left[H R_{N}^{n}\right]_{i}\right| e^{\gamma d x / 2} .
\end{aligned}
$$

Multiplying by $P_{i}$ and summing over all the $1 \leq i \leq d$, an estimate on $\mathcal{L}$ writes:

$$
\begin{aligned}
\mathcal{L}\left(R^{n+1}\right) \leq & (1-c \gamma d t) \mathcal{L}\left(R^{n}\right) \\
& -\sum_{i=1}^{d} P_{i}\left|R_{i, N}^{n+1}-R_{i, N}^{n}\right|\left(1-\frac{c \gamma d x}{v_{n u m}}\right) e^{-\gamma x_{N-1}} \\
& +\sum_{i=1}^{d} P_{i}\left|\left[H R_{N}^{n+1}\right]_{i}-\left[H R_{N}^{n}\right]_{i}\right| e^{\gamma d x / 2} .
\end{aligned}
$$

Using the definition of $|\cdot|_{1}$, the boundary terms can be compared with each others:

$$
\begin{aligned}
\mathcal{L}\left(R^{n+1}\right) \leq & (1-c \gamma d t) \mathcal{L}\left(R^{n}\right) \\
& +\left(\left|P H P^{-1}\right|{ }_{1} e^{\gamma d x / 2}-\left(1-\frac{c \gamma d x}{v_{\text {num }}}\right) e^{-\gamma x_{N-1}}\right) \sum_{i=1}^{d} P_{i}\left|R_{i, N}^{n+1}-R_{i, N}^{n}\right| .
\end{aligned}
$$


As $P \in D_{d}^{+}(\mathbb{R})$ is such that $\left|P H P^{-1}\right|_{1}<e^{-\gamma}$, we get for $\gamma d x$ sufficiently small (that is to say $\left.\gamma d x<\varepsilon\left(c, v_{\text {num }}, H\right)\right)$ :

$$
\mathcal{L}\left(R^{n+1}\right) \leq(1-c \gamma d t) \mathcal{L}\left(R^{n}\right)
$$

Summing with respect to time, one obtains:

$$
\mathcal{L}\left(R^{n+1}\right) \leq(1-c \gamma d t)^{n+1} \mathcal{L}\left(R^{0}\right)
$$

Using the fact that $1-x \leq e^{-x}$, the result of Lemma 3 is proved.

Remark 4 The rate of exponential convergence $\gamma c$ given by Theorem 3 is weaker than the one given in [13] for the continuous setting $\gamma v_{\min }$. This is mainly due to the choice of the limiter which guaranties only (19). To recover the rate of convergence from [13], one should impose:

$$
\nu v_{\min } \leq a_{n, j}^{i} \leq 1
$$

which is true for the upwind scheme but not for other limiters.

The following Theorem was already proved in [13]. The main difference here is that the proof comes from the convergence of our finite volume scheme.

Theorem 4 Under Hypothesis 2 and for all $P \in D_{d}^{+}(\mathbb{R}), \gamma>0$ such that $\left|P H P^{-1}\right|_{1}<$ $e^{-\gamma}$, there exists a constant $C(P, \gamma, H)$ depending only on $P, \gamma, H$ such that the entropy solution to (23) verifies

$$
\forall t \geq 0,\|R(t, \cdot)\|_{B V([0,1])} \leq C(P, \gamma, H) \exp \left(-\gamma v_{\min } t\right)\left\|R^{0}\right\|_{B V([0,1])}
$$

Proof For this proof, we take $\phi \equiv 0$ which corresponds to the upwind scheme. As a consequence, it is possible to take $v_{n u m}=v_{\min }$ in Hypothesis 1 . Then, it suffices to pass to the limit as $N$ goes to infinity in Lemma 3 using estimates (22), (21) and the equivalence between norms $\mathcal{L},\|\cdot\|_{B V([0,1])}($ see $(24))$.

\section{Simulations}

In this section, we illustrate numerically that slope limiter schemes are clearly less dissipative than the upwind scheme and capture better the behavior of the continuous solution. For computations, we consider the case where $d=4, \frac{d t}{d x}=0.4$ and the flux is given by:

$$
\forall 1 \leq i \leq 4, x \in \mathbb{R}, f_{i}\left(R_{i}\right)=0.5 R_{i}+\frac{0.5}{2^{i-1}} \arctan \left(R_{i}\right) .
$$

In such a case, $\min$ and $\max$ velocities are $v_{\min }=0.5$ and $v_{\max }=1$. Concerning the limiter, it is chosen as:

$$
\phi_{r}(r)=\max \{\min \{r, 1\}, 0\}, \psi_{d x}(v)= \begin{cases}v & \text { if }|v| \leq d x^{-\beta} \\ \operatorname{sign}(v) d x^{-\beta} & \text { otherwise }\end{cases}
$$

with $\beta=0.49$. This limiter is called a minmod limiter. 
If the focus is on the dissipativity of the scheme alone, a relevant boundary condition is:

$$
H=\left(\begin{array}{llll}
0 & 0 & 0 & 1 \\
1 & 0 & 0 & 0 \\
0 & 1 & 0 & 0 \\
0 & 0 & 1 & 0
\end{array}\right) .
$$

In such a case, the boundary condition does not dissipate energy. For a smooth initial data:

$$
R_{1}(t=0, .)=\cdots=R_{4}(t=0, .)=\cos (2 \pi x),
$$

we compare the $B V([0,1])$ norm of the solution given by the upwind scheme with the one given by the limiter one. It is expected that the limiter scheme is less dissipative than the upwind one when the solution is regular.

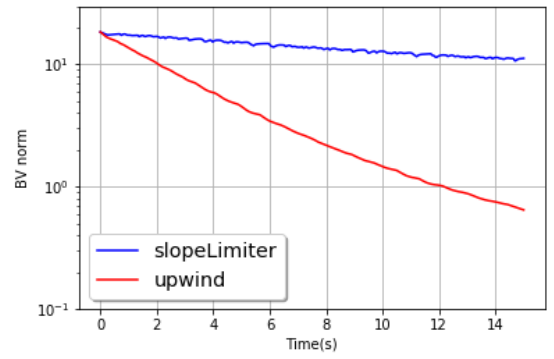

(a) $d t=10^{-2}$

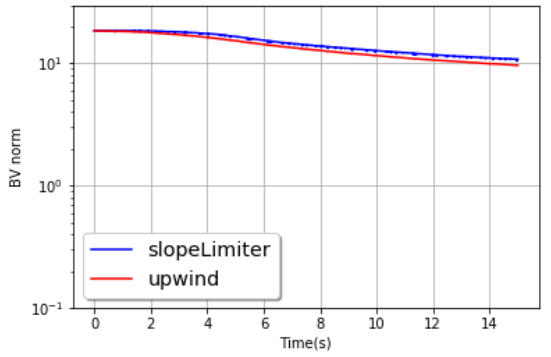

(b) $d t=5 \times 10^{-4}$

Fig. 3: The $B V$ norm of the solution for the non dissipative case

One sees in Figure 3a-3b that this is indeed the case. Obviously when the grid is fine enough, the dissipation created by the upwind scheme is negligible and results for both schemes are similar. Next, we can do the same analysis imposing this time, a dissipative feedback boundary condition:

$$
H=\left(\begin{array}{cccc}
0.5 & 0.1 & 0 & 0.1 \\
0.1 & 0.5 & 0.1 & 0 \\
0 & 0.1 & 0.5 & 0.1 \\
0.1 & 0 & 0.1 & 0.5
\end{array}\right)
$$

An optimization routine gives $\rho_{1}(H)=0.7$. Moreover, a less regular initial data is examined:

$$
\left\{\begin{array}{l}
R_{1}^{0}=x \geq 0.5 \\
R_{2}^{0}=-(x<0.5)+(x \geq 0.5) \\
R_{3}^{0}=x \geq 0.5 \\
R_{4}^{0}=-(x<0.5)+(x \geq 0.5)
\end{array}\right.
$$




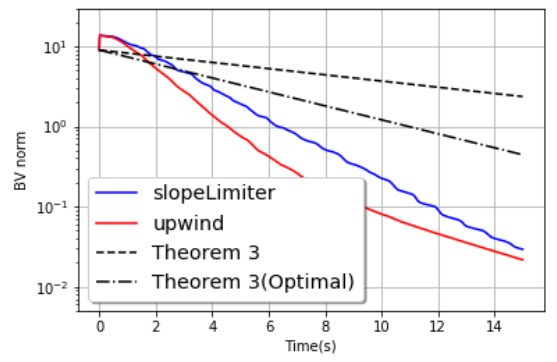

(a) $d t=10^{-2}$

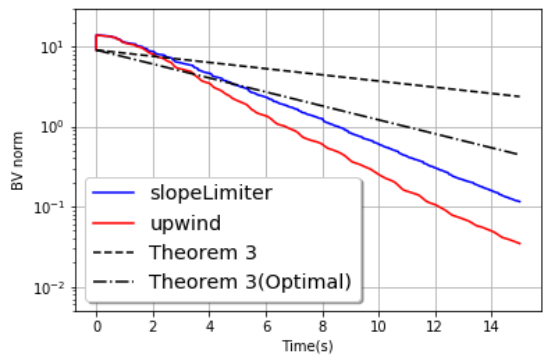

(b) $d t=5 \times 10^{-4}$

Fig. 4: The $B V$ norm of the solution for the dissipative case

The black dashed line corresponds to the estimation of the rate of convergence $\gamma v_{\text {num }}\left(v_{\text {num }}=0.5 v_{\min }, \gamma=-\log (0.7)\right.$ here $)$ given by Theorem 3 . We see this is not optimal since the rate of convergence is underestimated. Then, when the solution is small enough, it should be wise to take $v_{\min }=\min _{i} f_{i}^{\prime}(0)$ in Theorem 3 in order to estimate the rate of convergence when the solution is small (in $\left.L^{\infty}([0,1])\right)$. This rate is represented by the dashed dotted line for the upwind scheme $\left(v_{\text {num }}=v_{\text {min }}\right)$ in figures 4a-5b. This estimation fits better with the numerical experiments. With these results, one sees that Theorem 3 gives only a lower bound on the exponential convergence rate and not a precise estimation of it. Another remark to make is that the limiter scheme captures better the dissipation rate, the upwind scheme dissipating too much when the grid is coarse.

If we change the limiter, we have similar numerical results. For example, one can take the Van Leer limiter:

$$
\phi_{r}(r)=\frac{r+|r|}{1+|r|}
$$




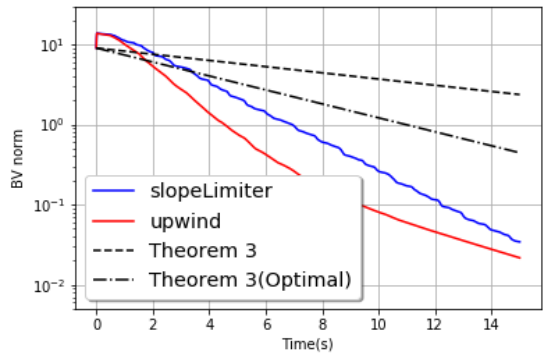

(a) $d t=10^{-2}$

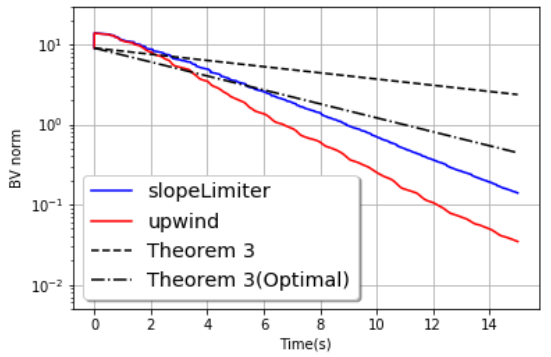

(b) $d t=5 \times 10^{-4}$

Fig. 5: The Van-Leer case

For completeness, we plot a part of the left trace of the solution for $t<15$ $\left(d t=5 \times 10^{-4}\right)$.

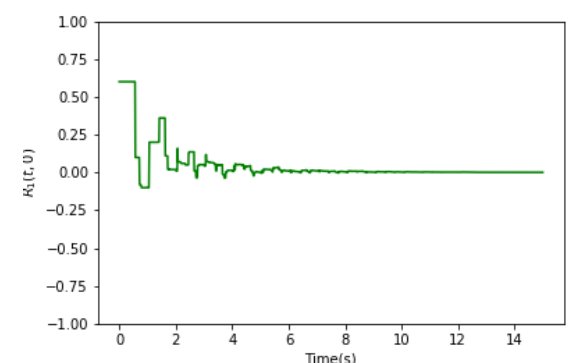

(a) $R_{1}(t, 0)$

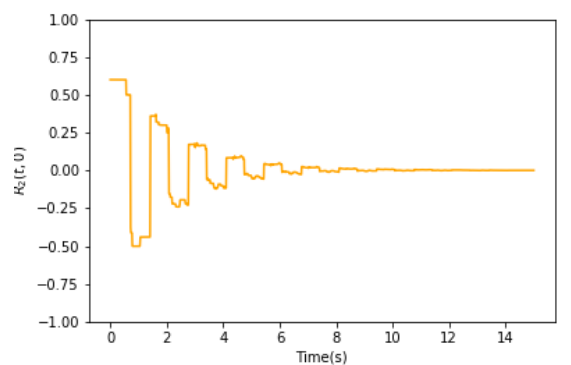

(b) $R_{2}(t, 0)$

Fig. 6: Trace of the solution

One clearly sees that the solution is rapidly damped when time evolves.

4.2 Comparison with the exact solution

An interesting analysis would be to compare convergence rates when it is possible to calculate the exact solution using the characteristics method. This is done by taking $d=2$ and a linear flux given by:

$$
\left\{\begin{array}{l}
f_{1}\left(R_{1}\right)=R_{1} \\
f_{2}\left(R_{2}\right)=1.6 \times R_{2}
\end{array}\right.
$$

The initial data is taken as follows: 


$$
\left\{\begin{array}{l}
R_{1}^{0}=\cos (2 \pi x) \\
R_{2}^{0}=\sin (2 \pi x)
\end{array}\right.
$$

For the non dissipative boundary condition, one takes:

$$
H=\left(\begin{array}{ll}
0 & 1 \\
1 & 0
\end{array}\right)
$$

One obtains Figure 7 where the energy dynamics are compared for both slope limiter and upwind schemes $\left(\frac{d t}{d x}=0.4\right)$ and also for the exact solution:

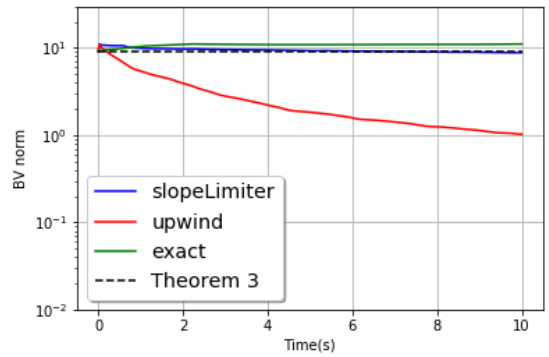

(a) $d t=10^{-2}$

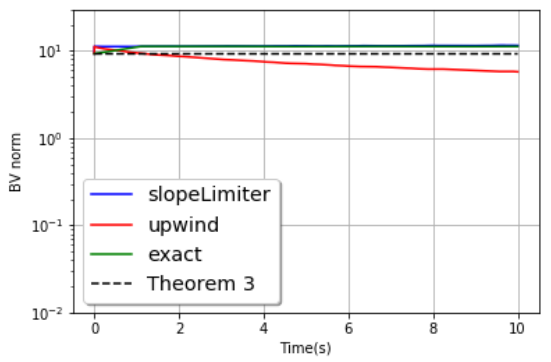

(b) $d t=10^{-3}$

Fig. 7: Non dissipative case: comparison with the exact solution

Note that the exact solution is calculated thanks to a recursive python routine that follows the characteristics backward in time. Additionally, the BV norm of the exact solution is calculated by sampling the solution uniformly, $E(1 / d x)$ times over the segment $[0,1]$.

For the dissipative boundary condition, one takes:

$$
H=\left(\begin{array}{cc}
0.2 & 0.5 \\
0.5 & 0.2
\end{array}\right)
$$

for which $\rho_{1}(H)=0.7$. In this case, the evolution of the $B V$ norm is represented in Figure 8 in the same way as in Figure 7. 


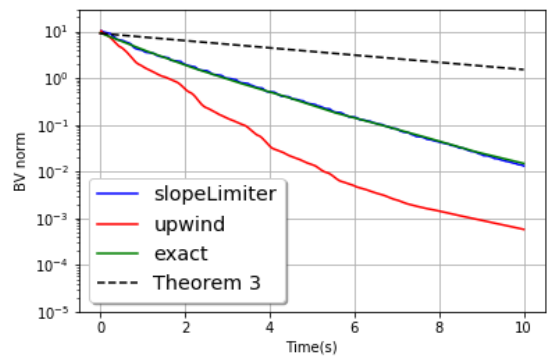

(a) $d t=10^{-2}$

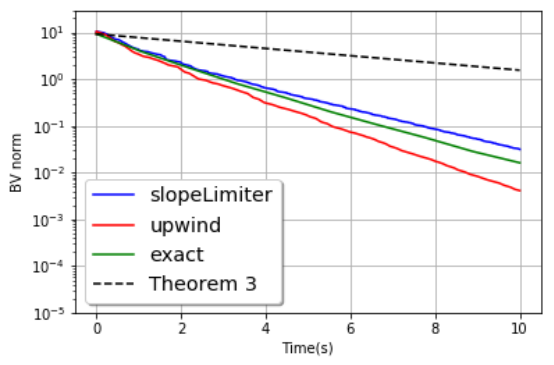

(b) $d t=10^{-3}$

Fig. 8: Dissipative case: comparison with the exact solution

For both dissipative and non dissipative cases, we can make the same conclusions as in section 4.1 in the sense that the upwind scheme dissipates too much when the grid is coarse whereas the slope limiter scheme fits better with the exact solution.

\subsection{Saturated control}

In this section, we analyze an example of system of scalar conservation laws for $d=2$ with saturated feedback control law. The same example as [13] is considered:

$$
\begin{cases}\partial_{t} R+\partial_{x}[f(R)] & =0 \\ R(t, 0) & =H R(t, 1)+B u(t) \\ R(0, x) & =R^{0}(x) \in B V([0,1])\end{cases}
$$

where:

$$
u(t)=\sigma(K R(t, 1)) .
$$

The operator $\sigma$ is the saturation by component with level of saturation equal to one:

$$
\forall 1 \leq i \leq 2, x \in \mathbb{R}, \begin{cases}\sigma_{i}(x)=x & \text { if }|x| \leq 1 \\ \sigma_{i}(x)=\operatorname{sign}(x) & \text { otherwise. }\end{cases}
$$

Matrices are defined as follows.

$$
H=\left(\begin{array}{cc}
0 & 1.1 \\
1 & 0
\end{array}\right), B=I_{2}, K=\left(\begin{array}{cc}
0 & -0.1050 \\
-0.1045 & 0
\end{array}\right) .
$$

As nonlinear flux, one takes $f(R)=\Lambda R+0.2\left(\arctan \left(R_{1}\right), \arctan \left(R_{2}\right)\right)$ with

$$
\Lambda=\left(\begin{array}{cc}
1 & 0 \\
0 & \sqrt{2}
\end{array}\right)
$$

For the scheme parameters, it is imposed that $d t=10^{-2}$ and $\nu=0.4$. Then, we take $R^{0}$ constant with value in $(-40,40)^{2}$ and look if the solution does not blow up 
at infinite time in $B V$ norm. In this way, it is possible to estimate the projection of the basin of attraction onto the space of constant initial data. Results are given below for the upwind and the minmod slope limiter scheme:

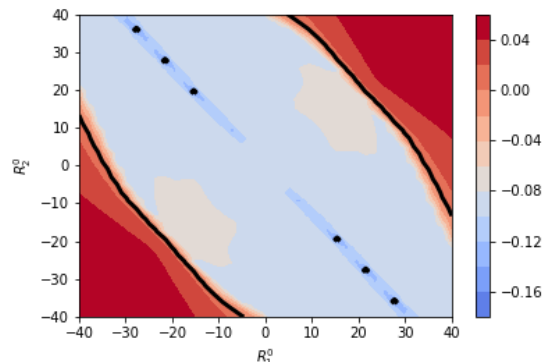

(a) Upwind

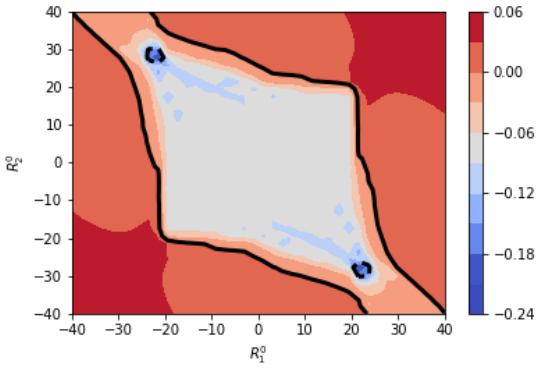

(b) Minmod limiter

Fig. 9: Comparison of basins of attraction $\left(d t=10^{-2}\right)$

The $x$-axis corresponds to the value of the first component of the initial data $R_{1}^{0}(x)=R_{1}^{0} \in \mathbb{R}$ whereas the $y$-axis corresponds to the value of the second component of the initial data $R_{2}^{0}(x)=R_{2}^{0} \in \mathbb{R}$. Contours correspond to the rate of exponential decay of the numerical solution for a time window of 50 seconds. If it is negative, the solution decays exponentially fast in BV norm. If it is positive, we have exponential divergence. The black thick contour corresponds to a dissipation rate equal to zero. Here, the same figure is plotted with a finer discretization $d t=10^{-3}$ for the minmod limiter scheme.

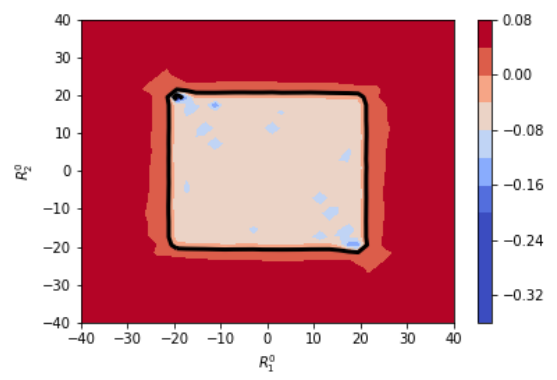

Fig. 10: Basin of attraction for $d t=10^{-3}$

We see that the upwind scheme overestimates the basin of attraction whereas it is not the case for the minmod slope limiter scheme. 


\section{Conclusion}

The exponential BV stability of general slope limiter scheme for system (23) has been established with an estimate of the dissipation rate. The main idea was to use Harten's formalism. Then, using new discrete Lyapunov techniques, we concluded on the exponential stability of the numerical solution in the spirit of the continuous case. The dissipation rate is estimated from below uniformly with respect to the discretization. Finally, the convergence of the scheme towards the entropy solution was established using the boundedness of $\psi_{d x}$.

There are some questions which remain open. The case of time dependent flux may not change the philosophy of the proof of exponential stability. However if we add a source term coupling the equations inside the domain the analysis is far from being obvious. Then the case where there are positive and negative characteristic velocities is not treated but this is only a question of change of variable. Finally combining both difficulties of velocities with different signs and the one of source terms, we know that a Lyapunov function does not exist in the continuous case [6]. It does not seem extravagant to suppose that such Lyapunov function does not exist in the discrete case. Backstepping techniques were designed to solve this difficulty when the flux is linear but it is not obvious that such methods can be directly applied to the corresponding numerical system.

\section{A Proof of Theorem 2}

The uniqueness can be proved by classical Kruzhkov techniques [13, Appendix B]. For the existence and the convergence result, it relies on classical techniques of $B V$ estimates based on the CFL condition (10). This is why we do not give any proof of these estimates and focus only on the effect of the boundary condition which is less classical. For more details, we refers to $[14]$.

Let $N$ be a positive integer and denote $R_{\Delta x}$ the numerical approximation given in Definition 1 . We recall that we defined $R_{0}^{n}$ as:

$$
R_{0}^{n}=g\left(R_{N}^{n}\right)
$$

\section{A.1 The $L^{\infty}$ estimate}

Now, define for all $n d t<\frac{\nu}{2}:=\tau$, the index $j_{\nu}(n)$ such that $\frac{2}{\nu}(n-1) d t \leq j_{\nu}(n) d x<\frac{2}{\nu} n d t$. From now on, we suppose that the time index $n$ verifies $n d t<\tau$. Under a CFL like condition (10), it is easy to prove that:

$$
\max _{\substack{n d t<\tau \\ j_{\nu}(n) \leq j \leq N}}\left|R_{j}^{n}\right| \leq \max _{0 \leq j \leq N}\left|R_{j}^{0}\right|
$$

and that:

$$
\max _{\substack{n d t<\tau \\ 0 \leq j \leq j_{\nu}(n)}}\left|R_{j}^{n}\right| \leq \max _{n d t<\tau}\left|R_{0}^{n}\right|+\max _{0 \leq j \leq N}\left|R_{j}^{0}\right| .
$$

Then, the Lipschitz character of the boundary condition allows to transform last inequality

$$
\max _{\substack{n d t<\tau \\ 0 \leq j \leq j_{\nu}(n)}}\left|R_{j}^{n}\right| \leq C\left(L_{g}\right) \max _{n d t<\tau}\left|R_{N}^{n}\right|+\max _{0 \leq j \leq N}\left|R_{j}^{0}\right|
$$

where $C\left(L_{g}\right)$ is a constant depending only on $L_{g}$. Finally, one can easily bound the left boundary term by the $L^{\infty}$ norm of the initial data to get: 


$$
\max _{\substack{n d t<\tau \\ 0 \leq j \leq j_{\nu}(n)}}\left|R_{j}^{n}\right| \leq\left(1+C\left(L_{g}\right)\right) \max _{0 \leq j \leq N}\left|R_{j}^{0}\right|
$$

Gathering (30) and (29), it holds:

$$
\left\|R_{\Delta x}\right\|_{L^{\infty}([0, \tau] \times[0,1])} \leq\left(1+C\left(L_{g}\right)\right)\left\|R_{\Delta x}^{0}\right\|_{L^{\infty}([0,1])} .
$$

Repeating this process, we obtain the following $L^{\infty}$ estimate for any $T>0$ :

$$
\left\|R_{\Delta x}\right\|_{L^{\infty}([0, T] \times[0,1])} \leq C\left(L_{g}, T\right)\left\|R_{\Delta x}^{0}\right\|_{L^{\infty}([0,1])} .
$$

where $C$ depends only on $T, L_{g}, \xi$ and $v_{\max }$. By the fact that $\left(R_{\Delta x}^{0}\right)_{N}$ is bounded in $L^{\infty}$ (recall that $R^{0}$ is in $B V([0,1])$ ), the $L^{\infty}$ estimate holds:

$$
\left\|R_{\Delta x}\right\|_{L^{\infty}([0, T] \times[0,1])} \leq C\left\|R^{0}\right\|_{L^{\infty}([0,1])}
$$

where $C$ depends only on $T, L_{g}, \xi$ and $v_{\max }$.

\section{A.2 The TV estimate}

We have,

$$
T V\left(R_{\bullet}^{n}\right)=T V_{\left[0, j_{\nu}\right]}\left(R_{\bullet}^{n}\right)+T V_{\left[j_{\nu}, N\right]}\left(R_{\bullet}^{n}\right) .
$$

Under the CFL condition (10), it is easy to prove that the first term on the left hand side of (32) can be bounded by a boundary term and an initial data term:

$$
T V_{\left[0, j_{\nu}\right]}\left(R_{\bullet}^{n}\right) \leq T V_{[0, n]}\left(R_{0}^{\bullet}\right)+T V\left(R_{\bullet}^{0}\right) .
$$

Moreover, by the Lipschitz character of the boundary condition, we get:

$$
T V_{\left[0, j_{\nu}\right]}\left(R_{\bullet}^{n}\right) \leq L_{g} T V_{[0, n]}\left(R_{N}^{\bullet}\right)+T V\left(R_{\bullet}^{0}\right) .
$$

Finally under (10), We can bound $T V_{[0, n]}\left(R_{N}^{\bullet}\right)$ by $T V\left(R_{\bullet}^{0}\right)$ to obtain:

$$
T V_{\left[0, j_{\nu}\right]}\left(R_{\bullet}^{n}\right) \leq\left(1+L_{g}\right) T V\left(R_{\bullet}^{0}\right) .
$$

Similarly, it is possible to prove that:

$$
T V_{\left[j_{\nu}, N\right]}\left(R_{\bullet}^{n}\right) \leq T V\left(R_{\bullet}^{0}\right) .
$$

and with (33), the following $T V$ estimate holds:

$$
\forall n d t<\tau, T V\left(R_{\bullet}^{n}\right) \leq C\left(L_{g}, T\right) T V\left(R_{\bullet}^{0}\right) .
$$

Repeating the process, one gets the following bound in $T V$ for $n d t<T$ with $T>0$ :

$$
T V\left(R_{\bullet}^{n}\right) \leq C\left(L_{g}, T\right) T V\left(R_{\bullet}^{0}\right)
$$

where $C\left(L_{g}, T\right)$ is a constant that depends solely on $L_{g}$ and $T$. By the fact that $\left(R_{.}^{0}\right)_{N}$ converges towards $R^{0}$ in $B V([0,1])$, one bounds the right hand side of last equation:

$$
\forall n d t \leq T, T V\left(R_{\bullet}^{n}\right) \leq C\left(L_{g}, T, R^{0}\right) .
$$


A.3 The $L^{1}([0,1])$ continuity estimate

For all integers $n>1$,

$$
\begin{aligned}
\sum_{j=0}^{N}\left|R_{j}^{n+1}-R_{j}^{n}\right| d x & =\sum_{j=1}^{N}\left|A_{j-1}^{n}\left(R_{j}^{n}-R_{j-1}^{n}\right)\right| d x+\left|R_{0}^{n+1}-R_{0}^{n}\right| d x \\
& \leq \sum_{j=1}^{N}\left|A_{j-1}^{n}\right|\left|R_{j}^{n}-R_{j-1}^{n}\right| d x+L_{g}\left|R_{N}^{n+1}-R_{N}^{n}\right| d x \\
& =\sum_{j=0}^{N-1}\left|A_{j}^{n}\right|\left|R_{j+1}^{n}-R_{j}^{n}\right| d x+L_{g}\left|A_{N-1}^{n}\left(R_{N}^{n}-R_{N-1}^{n}\right)\right| d x \\
& \leq\left(1+L_{g}\right) T V\left(R_{\bullet}^{n}\right) d x .
\end{aligned}
$$

By the triangle inequality, it holds for $n, m>0$

$$
\sum_{j=0}^{N}\left|R_{j}^{n+m}-R_{j}^{n}\right| d x \leq d x \sum_{l=n}^{n+m-1}\left(1+L_{g}\right) T V\left(R_{\bullet}^{l}\right) .
$$

And by the boundedness of the total variation (35), we get

$$
\sum_{j=0}^{N}\left|R_{j}^{n+m}-R_{j}^{n}\right| d x \leq C\left(L_{g},(n+m) d t, R^{0}\right) m d x .
$$

which is no more than:

$$
\left\|R_{\Delta x}((n+m) d t, \cdot)-R_{\Delta x}(n d t, \cdot)\right\|_{L^{1}([0,1])} \leq C\left(L_{g}, f,(n+m) d t, R^{0}\right) m d t .
$$

A.4 The $L_{l o c}^{1}\left(\mathbb{R}^{+}\right)$continuity estimate

We define for $T>0, n_{T}:=E(T / d t)$. For all integers $0 \leq j \leq N-1$, we have the following estimate:

$$
\begin{aligned}
\sum_{n=0}^{n_{T}}\left|R_{j+1}^{n}-R_{j}^{n}\right| d t & =\sum_{n=0}^{n_{T}} d t\left|\left(a_{j}^{n+1}\right)^{-1}\left(R_{j+1}^{n+1}-R_{j+1}^{n}\right)\right| \\
& \leq C d t \sum_{n=0}^{n_{T}}\left|R_{j+1}^{n+1}-R_{j+1}^{n}\right| .
\end{aligned}
$$

where we have used the fact that the $a_{i, j}^{n} \mathrm{~s}$ are bounded from below because of the Harten's condition (19). By the triangular inequality, it holds for $j_{0}<j_{1}$ :

$$
\sum_{n=0}^{n_{T}}\left|R_{j_{1}}^{n}-R_{j_{0}}^{n}\right| d t \leq C d t \sum_{j=j_{0}}^{j_{1}} T V_{\left[1, n_{T}+1\right]}\left(R_{j+1}^{\bullet}\right) .
$$

Similarly to proof of the boundedness of the space total variation (see (35)), we can prove the corresponding result for the time total variation. That is to say:

$$
\forall T>0,0 \leq j \leq N, T V_{\left[0, n_{T}\right]}\left(R_{j}^{\bullet}\right) \leq C\left(T, R^{0}, L_{g}\right) .
$$

Injecting this in (37), the following continuity estimate writes:

$$
\left\|R_{\Delta x}\left(\cdot, j_{1} d x\right)-R_{\Delta x}\left(\cdot, j_{0} d x\right)\right\|_{L^{1}([0, T])} \leq C\left(T, R^{0}, L_{g}, f\right)\left(j_{1}-j_{0}\right) d x .
$$




\section{A.5 Conclusion}

By Helly's Theorem, the sequence $\left(R_{\Delta x}\right)_{N}$ converges (up to a subsequence) in $L_{\text {loc }}^{1}\left(\mathbb{R}^{+} \times[0,1]\right)$ to a function $R \in L_{\text {loc }}^{1}\left(\mathbb{R}^{+}, B V([0,1])\right) \cap \operatorname{Lip} p_{l o c}\left(\mathbb{R}^{+}, L^{1}([0,1])\right)$. It remains to prove that $R$ is an entropy solution to (5).

Let $k \in \mathbb{R}^{d}$ and define $q_{k}(u):=f(u \top k)-f(k \perp u), \eta_{k}(u)=|u-k|$. The proof of the following result crucially depends on (17) and is given in a more general context in [10]. We give it here for completeness.

\section{Lemma 4 (Discrete entropy estimate)}

For all $k \in \mathbb{R}^{d}, N \in \mathbb{N}^{*}$, we have for all $n \in \mathbb{N}, 1 \leq j \leq N, 1 \leq i \leq d$ :

$$
\frac{1}{d t}\left(\left|R_{i, j}^{n+1}-k_{i}\right|-\left|R_{i, j}^{n}-k_{i}\right|\right)+\frac{1}{d x}\left(q_{k}\left(R_{i, j}^{n}+R_{i, j}^{\tilde{n}}\right)-q_{k}\left(R_{i, j-1}^{n}+R_{i, j-1}^{n^{\sim}}\right)\right) \leq C d x^{1-2 \beta} .
$$

In order to ease the reading, the $i$ index is dropped until the end of the proof. We take $\varphi \in C_{c}(] 0, T[\times] 0,1[)$. Multiplying (39) by $I_{j}^{n}(\varphi):=\int_{t^{n}}^{t^{n+1}} \int_{x_{j}-d x / 2}^{x_{j}+d x / 2} \varphi(t, x) d t d x$ and summing over $n, j$, one gets:

$\sum_{n=0}^{n_{T}} \sum_{j=1}^{N} \frac{\eta_{k}\left(R_{j}^{n+1}\right)-\eta_{k}\left(R_{j}^{n}\right)}{d t} I_{j}^{n}(\varphi)+\sum_{n=0}^{n_{T}} \sum_{j=1}^{N} \frac{q_{k}\left(R_{j}^{n}+\tilde{R}_{j}^{n}\right)-q_{k}\left(R_{j-1}^{n}+\tilde{R}_{j-1}^{n}\right)}{d x} I_{j}^{n}(\varphi) \leq C d x^{1-2 \beta}$.

A numerical integration by parts (owing the fact that $\varphi$ is zero at the boundary of $[0, T] \times[0,1]$ ), we get:

$$
\sum_{n=0}^{n_{T}} \sum_{j=1}^{N} \eta_{k}\left(R_{j}^{n}\right) \frac{I_{j}^{n-1}(\varphi)-I_{j}^{n}(\varphi)}{d t}+\sum_{n=0}^{n_{T}} \sum_{j=1}^{N} q_{k}\left(R_{j}^{n}+\tilde{R}_{j}^{n}\right) \frac{I_{j}^{n}(\varphi)-I_{j+1}^{n}(\varphi)}{d x} \geq C d x^{1-2 \beta}
$$

Passing to the limit owing that $\beta<1 / 2$, one gets

$$
\int_{0}^{T} \int_{0}^{1} \eta_{k}(R) \partial_{t} \varphi+q_{k}(R) \partial_{x} \varphi d x d t \geq 0 .
$$

\section{A.5.1 Boundary conditions and convergence estimates}

To finish the proof of the existence of a solution to (5), it remains to show that the initial and boundary conditions are verified in the almost everywhere sense. Let $\varepsilon>0$ and $0<s<1$

$$
\begin{aligned}
\left\|R(0, .)-R_{\Delta x}(0, .)\right\|_{L^{1}([0,1]) \leq} & \|R(0, .)-R(s, .)\|_{L^{1}([0,1])}+\left\|R(s, .)-R_{\Delta x}(s, .)\right\|_{L^{1}([0,1])} \\
& +\left\|R_{\Delta x}(s, .)-R_{\Delta x}(0, .)\right\|_{L^{1}([0,1])} \\
\leq & 2 C\left(g, R^{0}\right) s+\left\|R(s, .)-R_{\Delta x}(s, .)\right\|_{L^{1}([0,1])} .
\end{aligned}
$$

where we have used (36) to get the last inequality and the fact that $R \in \operatorname{Lip}_{\text {loc }}\left(\mathbb{R}^{+}, L^{1}([0,1])\right)$. Integrating with respect to $s$ on an interval $[0, t]$ for $0 \leq t \leq 1$, one gets

$$
\begin{aligned}
\left\|R(0, .)-R_{\Delta x}(0, .)\right\|_{L^{1}([0,1])} & \leq C\left(g, R^{0}\right) t+\frac{1}{t} \int_{0}^{t}\left\|R(s, .)-R_{\Delta x}(s, .)\right\|_{L^{1}([0,1])} d s \\
& \leq C\left(g, R^{0}\right) t+\frac{1}{t} \int_{0}^{1}\left\|R(s, .)-R_{\Delta x}(s, .)\right\|_{L^{1}([0,1])} d s
\end{aligned}
$$


Taking $t=\frac{\varepsilon}{2 C\left(g, R^{0}\right)}$ and $N$ sufficiently large such that $\int_{0}^{1}\|R(s, .)-R(s, .)\|_{L^{1}([0,1])} d s \leq$ $\frac{\varepsilon^{2}}{4 C\left(g, R^{0}\right)}$, one finally obtains:

$$
\left\|R(0, .)-R_{\Delta x}(0, .)\right\|_{L^{1}([0,1])} \leq \varepsilon .
$$

By the fact that $R_{\Delta x}^{0}$ converges towards $R^{0}$ in $L^{1}([0,1])$, we deduce that $R(0,)=.R^{0}$ in a $L^{1}$ sense and $R(0,)=.R^{0}$ almost everywhere.

Remark 5 The same procedure can be repeated for any $t \geq 0$ and prove (22).

Remark 6 We can use the same technique to prove that the boundary condition $R(t, 0)=$ $g(R(t, 1))$ is satisfied in the almost everywhere sense. In this case, the continuity estimate (38) is the key tool (see [13, p.24] for details).

The final step is to prove the convergence estimate (21). We give the sketch of the proof (see [13, p.24-25] for more details). In fact (21) is a consequence of Helly's Theorem applied to the sequence $\left(R_{\Delta x}\right)_{N>\tilde{N}}$ where $\tilde{N}>1$, on a time interval $[t, t+d t](d t>0)$. Then it suffices to take the limit when $\tilde{N}$ goes to infinity and when $d t$ goes to zero.

\section{References}

1. F. Alabau-Boussouira, Y. Privat, and E. Trélat. Nonlinear damped partial differential equations and their uniform discretizations. J. Funct. Anal., 273(1):352-403, 2017.

2. B. Andreianov, K.-H. Karlsen, and N.-H. Risebro. A theory of $L^{1}$-dissipative solvers for scalar conservation laws with discontinuous flux. Arch. Ration. Mech. Anal., 201(1):27-86, 2011.

3. B. P. Andreianov, G. M. Coclite, and C. Donadello. Well-posedness for vanishing viscosity solutions of scalar conservation laws on a network. Discrete Contin. Dyn. Syst., 37(11):5913-5942, 2017.

4. A. Aw and M. Rascle. Resurrection of "second order" models of traffic flow. SIAM J. Appl. Math., 60(3):916-938, 2000.

5. M. K. Banda and M. Herty. Numerical discretization of stabilization problems with boundary controls for systems of hyperbolic conservation laws. Math. Control Relat. Fields, 3(2):121-142, 2013.

6. G. Bastin and J.-M. Coron. On boundary feedback stabilization of non-uniform linear $2 \times 2$ hyperbolic systems over a bounded interval. Systems Control Lett., 60(11):900-906, 2011.

7. G. Bastin and J.-M. Coron. Stability and boundary stabilization of 1-D hyperbolic systems, volume 88 of Prog. in Nonlinear Differential Equations and their Applications. Birkhäuser/Springer, 2016.

8. P. Bernard and M. Krstic. Adaptive output-feedback stabilization of non-local hyperbolic PDEs. Automatica J. IFAC, 50(10):2692-2699, 2014.

9. A. Bressan. Hyperbolic systems of conservation laws, volume 20 of Oxford Lecture Series in Mathematics and its Applications. Oxford University Press, Oxford, UK, 2000. The one-dimensional Cauchy problem.

10. C. Chainais-Hillairet. Second-order finite-volume schemes for a non-linear hyperbolic equation: error estimate. Math. Methods Appl. Sci., 23(5):467-490, 2000.

11. J.-M. Coron, S. Ervedoza, S. S. Ghoshal, O. Glass, and V. Perrollaz. Dissipative boundary conditions for $2 \times 2$ hyperbolic systems of conservation laws for entropy solutions in BV. J. Differential Equations, 262(1):1-30, 2017.

12. J.-M. Coron and Z. Wang. Output feedback stabilization for a scalar conservation law with a nonlocal velocity. SIAM J. Math. Anal., 45(5):2646-2665, 2013.

13. M. Dus. Bv exponential stability for systems of scalar conservation laws using saturated feedback. preprint (to appear in SIAM SICON), 2020.

14. M. Dus. Exponential stability of a general slope limiter scheme for scalar conservation laws subject to a dissipative boundary condition. Preprint, January 2021. 
15. S. Ervedoza. Observability properties of a semi-discrete $1 \mathrm{D}$ wave equation derived from a mixed finite element method on nonuniform meshes. ESAIM Control Optim. Calc. Var., 16(2):298-326, 2010.

16. S. Ervedoza and E. Zuazua. Uniformly exponentially stable approximations for a class of damped systems. J. Math. Pures Appl. (9), 91(1):20-48, 2009.

17. R. Eymard, T. Gallouët, and R. Herbin. Finite volume methods. In Solution of Equation in $\mathbb{R}^{n}$ (Part 3), Techniques of Scientific Computing (Part 3), volume 7 of Handbook of Numerical Analysis, pages 713 - 1018. Elsevier, 2000.

18. S. Gerster and M. Herty. Discretized feedback control for systems of linearized hyperbolic balance laws. Math. Control Relat. Fields, 9(3):517-539, 2019.

19. A. Harten. High resolution schemes for hyperbolic conservation laws. Journal of Computational Physics, 49(3):357 - 393, 1983.

20. A. Hayat. On boundary stability of inhomogeneous $2 \times 21$-D hyperbolic systems for the $C^{1}$ norm. ESAIM Control Optim. Calc. Var., 25:31, 2019.

21. D. Kröner, S. Noelle, and M. Rokyta. Convergence of higher order upwind finite volume schemes on unstructured grids for scalar conservation laws in several space dimensions. Numer. Math., 71(4):527-560, 1995.

22. S. N. Kružkov. First order quasilinear equations with several independent variables. Mat. Sb. (N.S.), 81 (123):228-255, 1970.

23. R. J. LeVeque. Finite volume methods for hyperbolic problems. Cambridge Texts in Applied Mathematics. Cambridge University Press, Cambridge, 2002.

24. A. Marica and E. Zuazua. Localized solutions for the finite difference semi-discretization of the wave equation. C. R. Math. Acad. Sci. Paris, 348(11-12):647-652, 2010.

25. S. Osher and E. Tadmor. On the convergence of difference approximations to scalar conservation laws. Math. Comp., 50(181):19-51, 1988.

26. E. Yu. Panov. Existence of strong traces for generalized solutions of multidimensional scalar conservation laws. J. Hyperbolic Differ. Equ., 2(4):885-908, 2005.

27. P. K. Sweby. High resolution schemes using flux limiters for hyperbolic conservation laws. SIAM J. Numer. Anal., 21(5):995-1011, 1984.

28. L. R. Tébou and E. Zuazua. Uniform exponential long time decay for the space semidiscretization of a locally damped wave equation via an artificial numerical viscosity. $\mathrm{Nu}$ mer. Math., 95(3):563-598, 2003.

29. L. T. Tebou and E. Zuazua. Uniform boundary stabilization of the finite difference space discretization of the $1-d$ wave equation. Adv. Comput. Math., 26(1-3):337-365, 2007.

30. E. Trélat. Stabilization of Semilinear PDEs, and Uniform Decay under Discretization, page 31-76. London Mathematical Society Lecture Note Series. Cambridge University Press, 2017.

31. B. Van Leer. Towards the ultimate conservative difference scheme. V. A second-order sequel to Godunov's method [J. Comput. Phys. 32 (1979), no. 1, 101-136]. volume 135, pages 227-248. 1997. With an introduction by Ch. Hirsch, Commemoration of the 30th anniversary \{of J. Comput. Phys. \}.

32. A. Vasseur. Strong traces for solutions of multidimensional scalar conservation laws. Arch. Ration. Mech. Anal., 160(3):181-193, 2001.

33. E. Zuazua. Propagation, observation, and control of waves approximated by finite difference methods. SIAM Review, 47(2):197-243, 2005. 